\title{
Effect of Picrorrhiza Rhizoma on Dinitrofluorobenzene-induced Contact Dermatitis (Type I allergy)
}

\author{
Ji-Ha Park ${ }^{1}$, Sang-Nam LeE ${ }^{1}$, and Sae-Kwang Ku ${ }^{1,2,{ }^{*}}$ \\ ${ }^{1}$ College of Oriental Medicine, Daegu Haany University, Gyeongsan 712-715, Republic of Korea \\ ${ }^{2}$ Development Team for The New Drug of Oriental Medicine (BK21 program), Daegu Haany University, \\ Gyeongsan 712-715, Republic of Korea
}

(Received August 8, 2008; Revised August 28, 2008; Accepted September 6, 2008)

\begin{abstract}
The effect of Picrorrhiza Rhizoma (PR) aqueous extracts were evaluated on 2,4-dinitrofluorobenzene (DNFB)-induced contact dermatitis, type I allergic model. Contact dermatitis was induced by sensitization with dinitrophenyl-derivatized ovalbumin (DNP-OVA) and DNFB challenge as antigen. Three different concentrations of PR extracts $(300,150$ and $75 \mathrm{mg} / \mathrm{kg}$ ) were orally administered to DNP-OVA sensitization mice once a day for 7 days with reference materials; dexamethasone $(15 \mathrm{mg} / \mathrm{kg}$, intraperitoneal treatment). End of 7 days oral administration of PR extracts or intraperitoneal treatment of dexamethasone, the changes on the edematous changes and scratching behavior were measured. Immediate after DNFB challenge on ear or paw of DNP-OVA sensitized mice, increases of ear and paw thicknesses and weights were detected with anterior ear skin (dermis to epidermis) thickness and paw scratching behavior increases. However, these DNFB-induced increases on ear and paw thicknesses, weights and scratching behaviors were decreased by treatment of all three different dosages of PR extracts and dexamethasone, respectively. In addition, the increases of anterior skin thicknesses were also dramatically inhibited by treatment of all three different dosages of PR extracts and dexamethasone at histopathological observations. The results obtained in this study suggest that oral treatment of PR extracts also has relatively favorable effects on allergic dermatitis.
\end{abstract}

Key words: Picrorrhiza Rhizoma, Mouse, Contact dermatitis, 2,4-dinitrofluorobenzene, Atopy

\section{INTRODUCTION}

Until now, major types of hypersensitivities (allergies) have been divided in to IV types; Ig E mediated immdeiated type I, humoral antibody mediated cytotoxic type II, immune complex reactions activate inflammatory type III and T-lymphocytes mediated delayed type IV allergies (Nester et al., 1995). In cases of allergic disease with chronic and severe pruritus, such as contact dermatitis and urticaria, are accompanied by severe pruritus (Lorette and Vaillant, 1990; Klecz and Schwartz, 1992). Therefore, it is very important not only to treat the allergy but also to inhibit scratching of the lesion (Ishiguro et al., 2002). 2,4-dinitrofluorobenzene (DNFB) is used as an intermediate in the synthesis of pesticides. It is also used as a sensitizing agent and hapten in laboratory immunol-

\footnotetext{
${ }^{*}$ Corresponding author

Tel: +82-53-819-1549, Fax: +82-53-819-1269

E-mail: gucci200@hanmail.net
}

ogy and as a reagent to identify the terminal amino acids in a protein chain (Wang et al., 1998; Perez et al., 2004). The repeated application of DNFB to the ears of mice results in a typical allergic dermatitis and the simultaneous production of IgE antibody against DNFB (Nagai et al., 1997ab). Repeated application of DNFB onto the mouse ear causes the ear to swell accompanied by the rise of serum specific lgE levels (Nagai et al., 1997b). Thickening of epidermis, formation of scabs, and infiltration of abundant inflammatory cells are also induced (Ueda et al., 2003). Therefore DNFB-induced contact dermatitis model is a useful for evaluation of a potential treatment candidate for type I allergic dermatitis (Matsuda et al., 2002). The effects of a drug would be based on the ear swelling and incidence of scratching behaviors in this model (Matsuda et al., 2002).

Topical steroids have been a popular choice for treating various cutaneous disorders; however, the potential for significant local and systemic adverse events, like skin atrophy and hypothalamic-pituitary-adrenal axis suppres- 
sion, has limited their use (Gupta and Chow, 2004). Among glucocorticoids, dexamethasone is the one of widely used topical steroids on allergic dermatitis (Ganir et al., 1996; Matsumoto et al., 2005) and has been used as reference drug on develop of the new anti-allergic agents (Shichinohe et al., 1996; Ohtsuka et al., 2003).

A traditional Korean herbal medicine, Picrorrhiza Rhizoma (PR) is a dried root and stem of Picrorrhiza kurroa, and has been used as hepatoprotective agents such as jaundice. Until now, the nitric oxide scavenging activity (Jagetia and Baliga, 2004), cardioprotective effect (Senthil Kumar et al., 2001), anti-cancer effect (Jeena et al., 1999; Joy et al., 2000), anti-diabetic activity (Joy and Kuttan, 1999), anti-viral effect (Mehortra et al., 1990), hypolipidemic and hepatoprotective effects (Lee et al., 2006ab; Lee et al., 2008) of PR extracts have been evaluated. In addition, PR extracts also showed anti-inflammatory effects after oral administration (Lee and Ku, 2008). We hypothesized that that oral administration of PR extracts will be ameliorated the DNFB-induced contact dermatitis because this allergic dermatitis also showed quite similar phenomena like general inflammatory responses (Nagai et al., 1997ab; Ueda et al., 2003). When the surface of skin is inflamed, cracked or raw, many of these sting or burn when first applied of DNFB. This irritation will lessen as the allergic dermatitis improves related to anti-inflammation activity (Skinner, 2004; Rossetti et al., 2005). In the present study, the effects of PR aqueous extracts on the DNFB-induced contact dermatitis were monitored by comparing with dexamethasone, $15 \mathrm{mg} / \mathrm{kg}$, intraperitoneal treatment, according to previous methods (Watanabe et al., 1999; Matsuda et al., 2002).

\section{MATERIALS and Methods}

\section{Experimental Animals}

One hundred male ICR mice (6-wk old upon receipt, SLC, JAPAN) were used after acclimatization for 7 days. Animals were allocated five per polycarbonate cage in a temperature $\left(20-25^{\circ} \mathrm{C}\right)$ and humidity $(40-45 \%)$ controlled room. Light : dark cycle was $12 \mathrm{hr}: 12 \mathrm{hr}$ and feed (Samyang, Korea) and water were supplied free to access. About half animals are selected based on preliminary test (over 10\% increases of ear thickness) and each of 5 mice pre groups were used for ear edema test and paw scratching test.

\section{PR extracts}

The PR was purchased from Cho-Heung Pharmaceuti- cal Ind. Co. (Daegu, Korea) after confirming the morphology under microscopy. The voucher specimen has been deposited at Department of Herbal Biothechnology, Daegu Haany University (voucher number: DHU083-PR). The prepared PR (103 g) was boiled in 2 I of distilled water for $2 \mathrm{hrs}$ and filtrated. The filtrate was decompressed using a rotary vacuum evaporator (Lab. Camp, Korea) and lyophilized in a programmable freeze dryer (IIShin Lab., Korea). Total acquired PR extracts was $26.4 \mathrm{~g}$ (yield $25.65 \%$ ). Powders of PR extracts were stored in a desiccator to protect from light and moisture.

\section{Administration of drugs}

Three different concentrations of PR extracts $(300,150$ and $75 \mathrm{mg} / \mathrm{kg}$; dissolved in distilled water) were orally administered to DNP-OVA sensitization mice once a day for 7 days, and $15 \mathrm{mg} / \mathrm{kg}$ of dexamethasone-water soluble (Sigma, MO, USA; dissolved in saline) was intraperitoneal administered as same frequencies as PR extracts. In DNFB control, distilled water was orally administered instead of PR extracts as same methods.

\section{DNFB-induced contact dermatitis}

Dinitrophenyl-derivatized ovalbumin (DNP-OVA) was prepared according to the method described by Eisen and Belmam (1953) and was used as an antigen. DNPOVA were prepared by mixing 200mg OVA (Sigma, MO, USA) with $100 \mathrm{mg}$ 2,4-dinitrofluorobenzene (Sigma, MO, USA) for $18 \mathrm{~h}$ at room temperature. The number of DNP groups coupled to OVA was 3.2/molecule.

Animal selection: Mice were sensitized by an intraperitoneal injection of a mixture of DNP-OVA $(10 \mu \mathrm{g})$ and aluminum hydroxide gel $(1 \mathrm{mg})$ in saline $(0.2 \mathrm{ml})$. After one week, the mice were challenged by painting 10 il of $0.1 \%$ DNFB solution in ethanol on the inside and outside of the right and left ears as previously (Watanabe et al., 1999). About half animals showing an increment of percent change (over $15 \%$ ) of ear swelling were chosen

Sensitization: Next day of animal selection, the mice were again sensitized by the mixture of DNP-OVA (10 $\mathrm{mg}$ ) and aluminum hydroxide gel $(1 \mathrm{mg})$ in saline $(0.2 \mathrm{ml})$.

Antigen challenge: Challenged by painting 10il of $0.1 \%$ DNFB solution in ethanol on the inside of the right ears for ear swelling test, or on the hind paws of each mouse for scratching test.

\section{Changes in body weights}


Changes of body weights were calculated at second sensitization day and sacrifice with automatic electronic balance (Precisa Instrument, Switzland). In addition, body weight gains throughout experimental periods were also calculated, to reduce individual body weight differences from start of experiment.

\section{Ear and Paw thickness measurements}

The thicknesses of the intact and induced ear and/or paw were measured using an electronic digital caliper (Mytutoyo, JAPAN) $1 \mathrm{hr}$ after the DNFB challenge. The \% increment of thickness was expressed in percentage difference between the thickness of intact and induced sides and calculated as [(thicknesses of induced sides thicknesses of intact sides)/thicknesses of intact sides] $\times$ 100.

\section{Ear and Paw weight measurements}

$1 \mathrm{hr}$ after the DNFB challenge, circular sections of both sides of ear and paw rwere taken using a cork borer with a 7-mm diameter as previously described (Lee and $\mathrm{Ku}$, 2008), and weighed using an automatic electronic balance. The \% increment of weights was expressed in percentage difference between the weights of intact and induced sides and calculated as [(weights of induced sides - weights of intact sides)/weights of intact sides] $\times$ 100.

\section{Scratching behavior detection}

From immediately after DNFB challenges on the paw, the incidences of scratching behavior on the whole body and the site challenged with DNFB were counted for $1 \mathrm{hr}$. Behavior observation was done as previously method (Matsuda et al., 2002). In brief, after at least $1 \mathrm{hr}$ min acclimation to the experimental environment, mouse behaviors were monitored small windows served for counting the scratching. The scratching behavior was defined as movement of the hind limb excluding the movement for gait.

\section{Histopathology}

After measuring of thickness or weights, DNFB challenged ear was sampled and fixed in 10\% neutral buffered formalin after cross trimming. After paraffin embedding, 3-4 $\mu \mathrm{m}$ sections were prepared. Representative sections were stained with hematoxylin and eosin (H\&E) for light microscopical examination. After that the histological profiles of individual ear were evaluated.

Histomorphometry: The thicknesses of anterior skin parts - from epidermis to dermis (ear cartilages were excluded) were measured using automated CCD image analyzer (DMI-300 Image Processing; DMI, Korea) under magnification 100 of microscopy (Nikkon, Japan) at body regions of ear.

\section{Statistical analyses}

Multiple comparison tests for different dose groups were conducted. Variance homogeneity was examined using the Levene test. If the Levene test indicated no significant deviations from variance homogeneity, the obtain data were analyzed by one way ANOVA test followed by least-significant differences multi-comparison test to determine which pairs of group comparison were significantly different. In case of significant deviations from variance homogeneity were observed at Levene test, a nonparametric comparison test, Kruskal-Wallis $\mathrm{H}$ test was conducted. When a significant difference is detected in the Kruskal-Wallis $\mathrm{H}$ test, the Mann-Whitney U-Wilcoxon Rank Sum W test was conducted to determine the specific pairs of group comparison, which are significantly different. Statistical analyses were conducted using SPSS for Windows (Release 6.1.3., SPSS Inc., USA) and a $P$

Table I. Body weight and gains detected after oral treatment of PR extracts on DNFB-induced contact dermatitis mice

\begin{tabular}{cccc}
\hline \multirow{2}{*}{ Groups } & \multicolumn{2}{c}{ Body weights at } & \multicolumn{2}{c}{$\begin{array}{c}\text { Body weight gains } \\
(7 \text { days })\end{array}$} \\
\cline { 2 - 4 } & Start of treatment & Sacrifice & $1.43 \pm 0.60$ \\
DNFB-control & $41.08 \pm 2.15$ & $42.51 \pm 2.00$ & $-2.11 \pm 0.97^{*}$ \\
Dexamethasone & $41.01 \pm 1.70$ & $38.90 \pm 2.10^{*}$ & \\
PR extracts treated & & & $1.61 \pm 1.30$ \\
300mg/kg & $42.24 \pm 2.78$ & $43.85 \pm 3.04$ & $1.46 \pm 0.86$ \\
$150 \mathrm{mg} / \mathrm{kg}$ & $40.61 \pm 2.18$ & $42.07 \pm 2.21$ & $1.63 \pm 1.05$ \\
$75 \mathrm{mg} / \mathrm{kg}$ & $40.28 \pm 2.25$ & $41.91 \pm 2.44$ & \\
\hline
\end{tabular}

Values are expressed as Mean $\pm S D, g$ of ten mice; PR, Picrorrhiza Rhizoma; DNFB, 2,4-dinitrofluorobenzene; ${ }^{*} P<0.01$ as compared with DNFB control. 
Table II. Changes on the ear swelling after oral treatment of PR extracts in DNFB-induced contact dermatitis mice

\begin{tabular}{|c|c|c|c|}
\hline \multirow{2}{*}{ Groups } & \multicolumn{2}{|c|}{ Increment (\%) of ear ${ }^{\S}$} & \multirow{2}{*}{ Thickness of anterior skin (ìm) } \\
\hline & Thickness & Weights & \\
\hline DNFB-control & $63.46 \pm 25.18$ & $54.85 \pm 20.81$ & $727.661 \pm 141.308$ \\
\hline Dexamethasone & $18.85 \pm 10.05^{\star}$ & $14.60 \pm 12.98^{*}$ & $340.143 \pm 20.224^{*}$ \\
\hline \multicolumn{4}{|l|}{ PR extracts treated } \\
\hline $300 \mathrm{mg} / \mathrm{kg}$ & $21.45 \pm 12.00^{\star *}$ & $13.39 \pm 4.57^{*}$ & $399.952 \pm 54.312^{*}$ \\
\hline $150 \mathrm{mg} / \mathrm{kg}$ & $26.13 \pm 8.94^{\star *}$ & $15.53 \pm 9.87^{\star}$ & $504.853 \pm 87.898^{\star *}$ \\
\hline $75 \mathrm{mg} / \mathrm{kg}$ & $34.02 \pm 13.40$ & $9.95 \pm 6.64^{*}$ & $621.023 \pm 75.832$ \\
\hline
\end{tabular}

Values are expressed as Mean \pm SD of five mice; PR, Picrorrhiza Rhizoma; DNFB, 2,4-dinitrofluorobenzene; §Increment (\%) of paw was calculated as described in the Materials and methods section; ${ }^{*} \mathrm{P}<0.01$ and ${ }^{* *} \mathrm{P}<0.05$ as compared with DNFB control.

value $<0.05$ was considered significant.

\section{RESULTS}

\section{Changes on the body weights}

No meaningful changes on the body weight were detected in all tested groups as compared with DNFB control except for significant $(P<0.01)$ decrease in body weight and gains in dexamethasone treated group (Table I).

\section{Changes on the ear and paw thicknesses}

As results of ear and paw thickness measurements to observe the edematous changes, dramatical decreases of ear and paw thickness increments were detected in all tested groups as compared with DNFB control, respectively (Table II and III).

\section{Changes on the ear and paw weights}

To detect the inducement of edematous changes, the ear and paw weights were measured on individual mice in this study. As shown in Table and 2, significant $(P<0.01)$ decreases of ear and paw weight increments were detected in all tested groups as compared with DNFB control, respectively (Table II and III).

\section{Changes on the scratching numbers}

The effects of PR extracts on the pruritis were observed by measuring the frequency of scratching behaviors. In the present study, significant $(P<0.01)$ decreases of scratching behaviors were detected in all tested groups as compared with DNFB control (Table III).

\section{Histopathological changes}

Marked increases of skin thickness were detected in DNFB control with severe dermis edematous changes loosening of connective tissues at histopathological observations, but these histopathological changes were markedly inhibited by treatment of dexamethasone, PR extracts 300 and $150 \mathrm{mg} / \mathrm{kg}$ treated groups, respectively. In addition, they were also slightly reduced by treatment of PR extracts $75 \mathrm{mg} / \mathrm{kg}$ as compared with DNFB control (Fig. 1). The increases of skin thickness as detected by histomorphometry were detected in DNFB control due to severe dermis edematous changes, but these edematous changes were markedly inhibited by treatment of

Table III. Changes on the paw swelling and pruritis after oral treatment of PR extracts in DNFB-induced contact dermatitis mice

\begin{tabular}{cccc}
\hline \multirow{2}{*}{ Groups } & \multicolumn{2}{c}{ Increment (\%) of paw } & $\begin{array}{c}\text { Scratching behavior } \\
\text { (frequencies/hr) }\end{array}$ \\
\cline { 2 - 3 } & Thickness & Weights & $135.60 \pm 11.95$ \\
DNFB-control & $20.57 \pm 1.67$ & $10.22 \pm 2.89$ & $65.20 \pm 29.54^{*}$ \\
Dexamethasone & $3.06 \pm 0.92^{*}$ & $3.66 \pm 4.29^{*}$ & \\
PR extracts treated & & & $83.40 \pm 7.57^{*}$ \\
$300 \mathrm{mg} / \mathrm{kg}$ & $7.41 \pm 7.68^{*}$ & $2.51 \pm 1.19^{*}$ & $88.40 \pm 16.04^{*}$ \\
$150 \mathrm{mg} / \mathrm{kg}$ & $6.66 \pm 4.37^{*}$ & $2.97 \pm 2.83^{*}$ & $89.40 \pm 19.32^{*}$ \\
$75 \mathrm{mg} / \mathrm{kg}$ & $7.00 \pm 5.37^{*}$ & $3.87 \pm 2.96^{*}$ & \\
\hline
\end{tabular}

Values are expressed as Mean \pm SD of five mice; PR, Picrorrhiza Rhizoma; DNFB, 2,4-dinitrofluorobenzene; §Increment (\%) of ear was calculated as described in the Materials and methods section; ${ }^{*} P<0.01$ as compared with DNFB control 


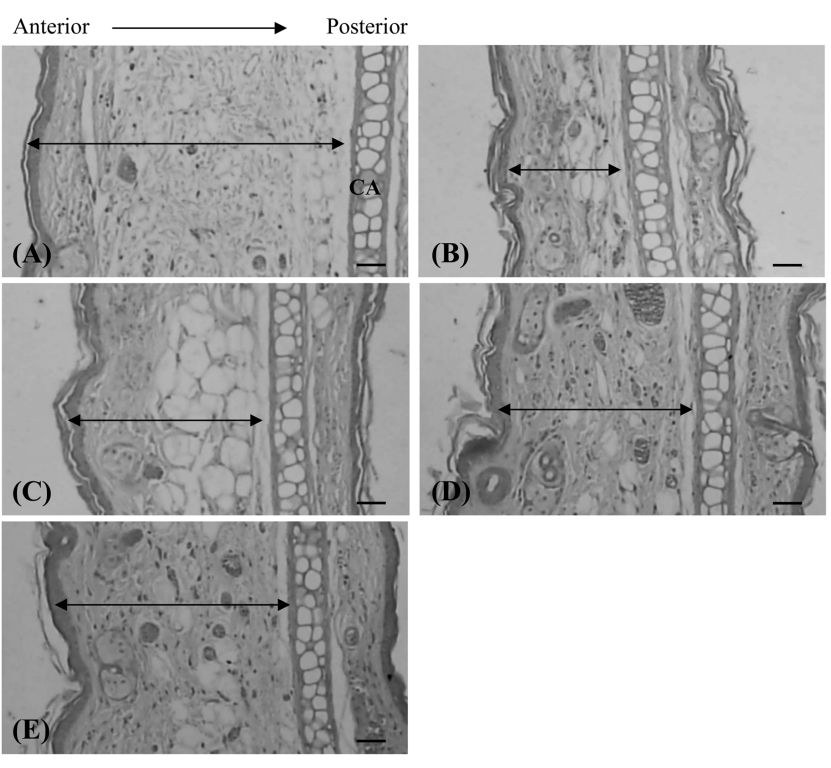

Fig. 1. Histopathological observations of ear in the DNFB control (A), Dexamethasone (B), PR extracts 300 (C), 150 (D) and 75 (E) $\mathrm{mg} / \mathrm{kg}$ treated groups.

Note that marked increases of skin thickness were detected in DNFB control with severe dermis edematous changes - loosening of connective tissues, but these increases on skin thicknesses were markedly inhibited by treatment of dexamethasone, PR extracts 300 and $150 \mathrm{mg} / \mathrm{kg}$ treated groups, respectively. In addition, they were also slightly reduced by treatment of PR extracts $75 \mathrm{mg} / \mathrm{kg}$ as compared with DNFB control; PR, Picrorrhiza Rhizoma; DNFB, 2,4-dinitrofluorobenzene; CA, ear cartilages; Arrows in figures means the anterior skin thicknesses measured in this study; All H\&E stain; Scale bars $=80 \mu \mathrm{m}$.

dexamethasone, all three different dosages of PR extracts (Table II).

\section{DISCUSSION}

Because the allergic dermatitis evoked by DNFB also showed quite similar phenomena like general inflammatory responses (Nagai et al., 1997ab; Ueda et al., 2003) and PR extracts also showed anti-inflammatory effects after oral administration (Lee and $\mathrm{Ku}, 2008$ ). Therefore, we considered that oral administration of PR extracts will be ameliorated the contact dermatitis. In the present study, contact dermatitis was induced by sensitization with DNP-OVA and DNFB challenge as antigen. Three different concentrations of PR extracts $(300,150$ and $75 \mathrm{mg} / \mathrm{kg}$ ) were orally administered to DNP-OVA sensitization mice once a day for 7 days with dexamethasone as reference. End of administration of PR extracts and dexamethasone, the changes on the edematous changes and scratching (pruritis) behavior were measured.
Immediate after DNFB challenge on DNP-OVA sensitized mice ear and paw, increases of ear and paw thickness and weights were detected with increases of anterior ear skin (dermis to epidermis) thickness and paw scratching behaviors. However, these edematous and allergic changes results from DNFB treatment were dramatically inhibited by 7 days pre-treatment of all three different dosages of PR extracts and dexamethasone in the present study.

The body weight decreases detected in dexamethasone treated group were considered as direct toxicity of glucocorticoid, steroids have been a popular choice for treating various cutaneous disorders; however, the potential for significant local and systemic adverse events, like skin atrophy and HPA axis suppression, has limited their use (Ganir et al., 1996; Gupta and Chow, 2004; Matsumoto et al., 2005). Anyway, no meaningful changes on the body weights were detected by treatment of all three different dosages of PR extracts as compared with DNFB control, respectively.

Repeated application of DNFB onto the mouse ear causes swell accompanied by the rise of serum specific lgE levels (Nagai et al., 1997b). Thickening of epidermis and infiltrations of inflammatory cells are also evoked (Ueda et al., 2003). Therefore DNFB-induced contact dermatitis model has been used for evaluation of a potential treatment candidate for type I allergic dermatitis (Matsuda et al., 2002). DNFB to mice sensitized by DNPOVA caused edemas similar to an occurrence of allergic dermatitis is IgE antibody dependent appearing $1 \mathrm{~h}$ after application of DNFB (Watanabe et al., 1999). In the present study, marked edematous changes, increment of thickness and weights were detected on DNFB challenged ear and paw with pruritis. However, these allergic changes induced by DNFB challenges were markedly inhibited by administration of all three dosages of PR extracts. It is considered as direct evidences that PR extracts can be ameliorated the type I allergic dermatitis, and these are considered as result of anti-inflammatory effects related to antioxidant effects of PR extracts already reported (Lee and $\mathrm{Ku}, 2008$ ). The main active compounds of PR extracts showed ameliorate effects of on the DNFB-induced dermatitis in the present study are considered as picroside and apocynin because it has been reported that picroside and apocynin showed various pharmacological effects including anti-oxidant effects (Joy et al., 2000)

\section{REFERENCES}

Eisen, H.N. and Belmam, S. (1953). Studies of hypersensitivity 
to low molecular weight substances. II. Reactions of some allergenic substituted dinitrobenzenes with cysteine or cystine of skin proteins. J. Exp. Med. 98, 533-549.

Ganir, E.M., Capulong, M.C., Tahara, K., Akasawa, A. and likura, Y. (1996). Treatment of atopic dermatitis in children: the importance of skin care and environmental control. Acta Paediatr. Jpn. 38, 702-704.

Gupta, A.K. and Chow, M. (2004). Prednicarbate (Dermatop): profile of a corticosteroid. J. Cutan. Med. Surg. 8, 244-247.

Ishiguro, K., Oku, H., Suitani, A. and Yamamoto, Y. (2002). Effects of conjugated linoleic acid on anaphylaxis and allergic pruritus. Biol. Pharm. Bull. 25, 1655-1657.

Jagetia, G.C. and Baliga, M.S. (2004). The evaluation of nitric oxide scavenging activity of certain Indian medicinal plants in vitro: a preliminary study. J. Med. Food 7, 343-348.

Klecz, R.J. and Schwartz, R.A. (1992). Pruritus. Am. Family Physician 45, 2681-2686.

Jeena, K.J., Joy, K.L. and Kuttan, R. (1999). Effect of Emblica officinalis, Phyllanthus amarus and Picrorrhiza kurroa on Nnitrosodiethylamine induced hepatocarcinogenesis. Cancer Lett. 136, 11-16.

Joy, K.L. and Kuttan, R. (1999). Anti-diabetic activity of Picrorrhiza kurroa extract. J. Ethnopharmacol. 67, 143-148.

Joy, K.L., Rajeshkumar, N.V., Kuttan, G. and Kuttan, R. (2000). Effect of Picrorrhiza kurroa extract on transplanted tumours and chemical carcinogenesis in mice. J. Ethnopharmacol. 71, 261-266.

Lee, H.S., Ahn, H.C. and Ku, S.K. (2006a). Hypolipemic effect of water extracts of Picrorrhiza rhizoma in PX-407 induced hyperlipemic ICR mouse model with hepatoprotective effects: A prevention study. J. Ethnopharmacol. 105, 380-386.

Lee, H.S. and Ku, S.K. (2008). Effects of Picrorrhiza Rhizoma on acute inflammation in mice. Biomolecules \& Therapeutics, 16, $137-140$

Lee, H.S., Woo, S.J. and Ku, S.K. (2008). Hypolipemic and hepatoprotective effects of picrorrhiza rhizome in high fat diet supplied mice. A prevention study. Biomolecules \& Therapeutics 16, 46-53.

Lee, H.S., Yoo, C.B. and Ku, S.K. (2006b). Hypolipemic effect of water extracts of Picrorrhiza rhizoma in high fat diet treated mouse. Fitoterapia, 77, 579-584.

Lorette, G. and Vaillant, L. (1990). Pruritus: current concepts in pathogenesis and treatment. Drugs 39, 218-223.

Matsuda, H., Tomohiro, N., Ido, Y. and Kubo, M. (2002). Antiallergic effects of cnidii monnieri fructus (dried fruits of Cnidium monnieri) and its major component, osthol. Biol. Pharm. Bull. 25, 809-812.

Matsumoto, K., Mizukoshi, K., Oyobikawa, M., Ohshima, H., Sakai, Y. and Tagami, H. (2005). Objective evaluation of the efficacy of daily topical applications of cosmetics bases using the hairless mouse model of atopic dermatitis. Skin Res. Technol. 11, 209-217.

Mehrotra, R., Rawat, S., Kulshreshtha, D.K., Patnaik, G.K. and Dhawan, B.N. (1990). In vitro studies on the effect of certain natural products against hepatitis B virus. Indian J. Med. Res. 92, 133-138.

Nagai, H., Hiyama, H., Matsuo, A., Ueda, Y., Inagaki, N. and Kawada, K. (1997a). FK-506 and cyclosporin A potentiate the $\lg \mathrm{E}$ antibody production by contact sensitization with hapten in mice. J. Pharmacol. Exp. Ther. 283, 321-327.

Nagai, H., Matsuo, A., Hiyama, H., Inagaki, N. and Kawada, K. (1997b). Immunoglobulin $\mathrm{E}$ production in mice by means of contact sensitization with a simple chemical, hapten. J. Allergy Clin. Immunol. 100, S39-44.

Nest, E.W., Roberts, C.E. and Nester, M.T. (1995). Microbiology, a human perspects. Wm. C. Brown Communications, Inc., Dubuque.

Ohtsuka, E., Kawai, S., Nojima, H., Andoh, T., Kamimura, K. and Kuraishi, Y. (2003). Inhibitory effect of azelastine on allergic itch-associated response in mice sensitized with mosquito salivary glands extract. J. Pharmacol. Sci. 91, 263-266.

Perez, A., Narayan, S. and Sansom, J. (2004). Occupational contact dermatitis from 2,4-dinitrofluorobenzene. Contact Dermatitis 51, 314.

Rossetti, G. Laffitte, E., Eigenmann, P.A., Lubbe, J., Hohl, D. and Hofer, M.F. (2005). Treatment of atopic dermatitis: practical approach. Rev. Med. Suisse 1, 501-504

Senthil Kumar, S.H., Anandan, R., Devaki, T. and Santhosh Kumar, M. (2001). Cardioprotective effects of Picrorrhiza kurroa against isoproterenol-induced myocardial stress in rats. Fitoterapia 72, 402-405.

Shichinohe, K., Shimizu, M. and Kurokawa, K. (1996). Effect of M-711 on experimental skin reactions induced by chemical mediators in rats. J. Vet. Med. Sci. 58, 419-423.

Skinner, R. (2004). Role of topical therapies in the management of cutaneous disease. J. Cutan. Med. Surg. 8, 22-31.

Ueda, Y., Sone, T., Inagaki, N. and Nagai, H. (2003). Effects of prednisolone on the cutaneous reaction and skin barrier function in mice treated with a hapten. Biol. Pharm. Bull. 26, 618621.

Wang, H.W., Tedla, N., Lloyd, A.R., Wakefield, D. and McNeil, P.H. (1998). Mast cell activation and migration to lymph nodes during induction of an immune response in mice. $J$. Clin. Invest. 102, 1617-1626.

Watanabe, C., Satoh, T., Tahara, E., Murakami, K., Hayashi, K., Hase, K., Andoh, T., Kuraishi, Y., Kadota, S., Nagai, H. and Saiki I. (1999). Inhibitory mechanisms of glycoprotein fraction derived from Miscanthus sinensis for the immediate phase response of an lgE-mediated cutaneous reaction. Biol. Pharm. Bull. 22, 26-30. 